Federal Reserve Bank of Dallas

Globalization and Monetary Policy Institute

Working Paper No. 9

http://www.dallasfed.org/assets/documents/institute/wpapers/2008/0009.pdf

\title{
Country Portfolios in Open Economy Macro Models*
}

\author{
Michael B. Devereux \\ University of British Columbia \\ CEPR \\ Globalization and Monetary Policy Institute, Federal Reserve Bank of Dallas \\ Alan Sutherland \\ University of St Andrews \\ CEPR
}

April 2008

\begin{abstract}
This paper develops a simple approximation method for computing equilibrium portfolios in dynamic general equilibrium open economy macro models. The method is widely applicable, simple to implement, and gives analytical solutions for equilibrium portfolio positions in any combination or types of asset. It can be used in models with any number of assets, whether markets are complete or incomplete, and can be applied to stochastic dynamic general equilibrium models of any dimension, so long as the model is amenable to a solution using standard approximation methods. We first illustrate the approach using a simple two-asset endowment economy model, and then show how the results extend to the case of any number of assets and general economic structure.
\end{abstract}

JEL codes: E52, E58, F41

\footnotetext{
* Michael B. Devereux, Department of Economics, University of British Columbia, 997-1873 East Mall, Vancouver, B.C. Canada V6T 1Z1. devm@interchange.ubc.ca. Alan Sutherland, School of Economics and Finance, University of St Andrews, St Andrews, Fife, KY16 9AL, UK. ajs10@st-and.ac.uk. We are grateful to Philip Lane, Klaus Adam, Pierpaolo Benigno, Gianluca Benigno, Berthold Herrendorf, Fabrizio Perri, Robert Kollmann, Giancarlo Corsetti, Morten Ravn, Martin Evans and Viktoria Hnatkovska for comments on an earlier draft of this paper. This research is supported by the ESRC World Economy and Finance Programme, award number 156-25-0027. Devereux also thanks SSHRC, the Bank of Canada, and the Royal Bank of Canada for financial support. The views in this paper are those of the authors and do not necessarily reflect the views of the Federal Reserve Bank of Dallas or the Federal Reserve System.
} 


\section{Introduction}

This paper develops a simple and tractable approach to computing equilibrium financial asset portfolios in open economy dynamic stochastic general equilibrium (DSGE) models. To a large extent, existing open economy macroeconomic models ignore portfolio composition, analyzing financial linkages between countries in terms of net foreign assets, with no distinction made between assets and liabilities. But recent research has highlighted the presence of large cross-country gross asset and liability positions, and considerable heterogeneity among countries in portfolio composition among different classes of assets. Lane and Milesi-Ferretti (2001, 2006) show that these gross portfolio holdings have grown rapidly, particularly in the last decade. Their measures show that even large countries such as the UK hold gross assets and liabilities that are multiples of GDP.

The growth in international financial portfolios raises a number of important questions for open economy macroeconomics. What are the determinants of the size and composition of gross portfolio positions? Can standard theories account for the observed structure of portfolio holdings? Moreover, the large size of gross positions makes it likely that the portfolio composition itself affects macroeconomic outcomes. With gross positions as large as GDP, unanticipated changes in exchange rates or asset prices can generate valuation effects that are the same order of magnitude as annual current accounts ${ }^{1}$. This raises questions about how portfolio composition may affect the international business cycle and international transmission of shocks. Finally, by generating significant wealth re-distributions in response to fluctuations in exchange rates and asset prices, international portfolio composition may have significant implications for economic policy. How should monetary and fiscal policies be designed in an environment of endogenous portfolio choice?

While these questions are obviously of interest to open economy macroeconomists and policymakers, current theoretical models and solution methods cannot answer them in any very systematic way. This is because the standard approaches to solving general equilibrium models make it difficult to incorporate portfolio choice. The usual method of analysis in DSGE models is to take a linear approximation around a non-stochastic steady

\footnotetext{
${ }^{1}$ Lane and Milesi-Ferretti (2001) emphasize the quantitative importance of valuation effects on external assets and liabilities. See also subsequent work by Ghironi et al. (2005), Gourinchas and Rey (2005), and Tille (2003, 2004).
} 
state. But optimal portfolios are not uniquely defined in a non-stochastic steady state, so there is no natural point around which to approximate. Moreover, portfolios are also not defined in a first-order approximation to a DSGE model, since such an approximation satisfies certainty equivalence, so all assets become perfect substitutes. As a result, the analysis of portfolio choice in DSGE models appears to be intractable in all but the most restricted of cases. ${ }^{2}$

In this paper we develop and present an approximation method which overcomes these problems. Our method can be applied to any standard open economy model with any number of assets, any number of state variables, and complete or incomplete markets, so long as the model is amenable to solution by the usual approximation methods. We find a general formula for asset holdings which can be very easily incorporated into the standard solution approach for DSGE models. The technique is simple to implement and can be used to derive either analytical results (for sufficiently small models) or numerical results for larger models.

A key feature of our approach is to recognize that, at the level of approximation usually followed in open economy macroeconomics, one only requires a solution for the 'steady-state' portfolio holdings. The steady state portfolio is defined as the constant (or 'zero-order' ) term in a Taylor series approximation of the true equilibrium portfolio function. Higher-order aspects of portfolio behaviour are not relevant for first-order accurate macro dynamics. Equivalently, time variation in portfolios is irrelevant for all questions regarding first-order responses of macroeconomic variables like consumption, output, real exchange rates, etc. in a DSGE model. Therefore, the solution we derive exhausts all the macroeconomic implications of portfolio choice at this level of approximation.

How do we obtain the zero-order component of the equilibrium portfolio? We do so

\footnotetext{
${ }^{2}$ If there are enough financial assets to allow perfect risk sharing (so that international financial markets are effectively complete) then the problem becomes somewhat easier. In this case, it is possible to identify an equilibrium macroeconomic allocation independent of financial structure, and then, given this allocation, one can derive the implied portfolios which support the equilibrium. Engel and Matsumoto (2005) and Kollmann (2006) represent examples of such an approach. However, when markets are incomplete (in the sense that there are not sufficient assets to allow perfect risk sharing) optimal portfolios and macroeconomic equilibrium must be derived simultaneously. This makes the problem considerably more difficult. Heathcote and Perri (2004) provide one example of an incomplete markets model in which it is possible to derive explicit expressions for equilibrium portfolios. Their model is, however, only tractable for a specific menu of assets and for specific functional forms for preferences and technology.
} 
using a combination of a second-order approximation of the portfolio selection condition with a first-order approximation to the remaining parts of the model. Of course, these two approximations will be interdependent; the endogenous portfolio weights will depend on the variance-covariance matrix of excess returns produced by the general equilibrium model, but that in turn will depend on the portfolio positions themselves. We show that this simultaneous system can be solved to give a simple closed-form analytical solution for the equilibrium portfolio.

While our solution procedure is novel, the mathematical foundations of the solution we derive are already established in the literature, in particular in the work of Samuelson (1970), and in different form by Judd (1998) and Judd and Guu (2001). Samuelson shows how a mean-variance approximation of a portfolio selection problem is sufficient to identify the optimal portfolio in a near-non-stochastic world. In a related paper, Judd and Guu show how the same equilibrium can be identified by using a combination of a Bifurcation theorem and the Implicit Function Theorem. Our solution approach relies on first-order and second-order approximations of the model, rather than the Implicit Function and Bifurcation Theorems, but the underlying theory described by Judd and Guu (2001) is applicable to our equilibrium solution. In particular, the steady-state portfolio derived using our technique corresponds to a bifurcation point in the set of nonstochastic equilibria. The main contribution of this paper is to show how this solution can easily be derived in standard DSGE models. We note in addition, that there is nothing about the approximation method that restricts its use to open economy models. It can be applied to any heterogeneous agent DSGE model, whether in a closed or open economy context. ${ }^{3}$

As we have already stated, the steady-state portfolio is all that is needed in order to analyze the first-order properties of a general equilibrium model. But for many purposes, it may be useful to analyze the dynamics of portfolio holdings themselves. In addition, in order to do welfare analysis, it is usually necessary to analyze a second-order approximation of a model. At the level of second-order approximation, time variation in portfolios becomes relevant for macroeconomic dynamics. But these features can be obtained by an extension of our method to higher-order approximations of the model. In

\footnotetext{
${ }^{3}$ Samuelson (1970) and Judd and Guu (2001) did not develop their results in open economy (or general equilibrium) contexts.
} 
particular, the state-contingent, or first-order aspects of the equilibrium portfolio, can be obtained by combining a third-order approximation of the portfolio selection equations, with a second-order approximation to the rest of the model. The current paper focuses on the derivation of steady-state portfolios because this represents a distinct and valuable first-step in the analysis of portfolio choice in open-economy DSGE models. We do, however, discuss briefly the extension of the method to higher orders. In a companion paper, Devereux and Sutherland (2007), we show how higher-order solutions to portfolios also have an analytical representation.

In the related literature a number of approaches have been developed for analysing portfolio choice in incomplete-markets general equilibrium models. In a recent paper, Tille and Van Wincoop (2007) show how the zero and higher-order components of portfolio behaviour in an open economy model can be obtained numerically via an iterative algorithm. Their approach delivers a numerical solution for steady-state portfolios in manner analogous to the analytical solutions derived in this paper. Judd et al (2002) develop a numerical algorithm based on 'spline collocation' and Evans and Hnatkovska (2005) present a numerical approach that relies on a combination of perturbation and continuoustime approximation techniques. ${ }^{4}$ The methods developed by Judd et al and Evans and Hnatkovska are very complex compared to our approach and they represent a significant departure from standard DSGE solution methods. Devereux and Saito (2005) use a continuous time framework which allows some analytical solutions to be derived, but their approach can not handle general international macroeconomic models with diminishingreturns technology or sticky nominal goods prices.

This paper proceeds as follows. The next section sets out a two-asset portfolio choice problem within a simple two-country endowment model and shows how our method can be applied in this context. Section 3 develops a more general n-asset portfolio problem within a generic two country DSGE model and shows how the method can be generalised to accommodate a wide class of models. Section 4 briefly outlines how the method can be extended to derive a solution for the first-order component of the equilibrium portfolio. Section 5 concludes the paper.

\footnotetext{
${ }^{4}$ Evans and Hnatkovska (2005) develop an approach similar to that of Campbell and Viceira (2005), who present a comprehensive analysis of optimal portfolio allocation for a single agent.
} 


\section{Example: A Simple Two-Asset Endowment Model}

\subsection{The Model}

We first illustrate how the solution procedure works in a simple two-country example with only two internationally traded assets, where agents consume an identical consumption good, and income takes the form of a exogenous endowment of the consumption good.

Agents in the home country have a utility function of the form

$$
U_{t}=E_{t} \sum_{\tau=t}^{\infty} \beta^{\tau-t} u\left(C_{\tau}\right)
$$

where $C$ is consumption and $u\left(C_{\tau}\right)=\left(C_{\tau}^{1-\rho}\right) /(1-\rho)$.

The budget constraint for home agents is given by

$$
\alpha_{1, t}+\alpha_{2, t}=\alpha_{1, t-1} r_{1, t}+\alpha_{2, t-1} r_{2, t}+Y_{t}-C_{t}
$$

where $Y$ is the endowment received by home agents, $\alpha_{1, t-1}$ and $\alpha_{2, t-1}$ are the real holdings of the two assets (purchased at the end of period $t-1$ for holding into period $t$ ) and $r_{1, t}$ and $r_{2, t}$ are gross real returns. It is assumed that the vector of available assets is exogenous and predefined. The stochastic process determining endowments and the nature of the assets and the properties of their returns are specified below.

Define $W_{t}=\alpha_{1, t}+\alpha_{2, t}$ to be the total net claims of home agents on the foreign country at the end of period $t$ (i.e. the net foreign assets of home agents). The budget constraint can then be re-written as

$$
W_{t}=\alpha_{1, t-1} r_{x, t}+r_{2, t} W_{t-1}+Y_{t}-C_{t}
$$

where

$$
r_{x, t}=r_{1, t}-r_{2, t}
$$

Here asset 2 is used as a numeraire and $r_{x, t}$ measures the "excess return" on asset 1.

At the end of each period agents select the portfolio of assets to hold into the following period. Thus, for instance, at the end of period $t$ home agents select $\alpha_{1, t}$ to hold into period $t+1$. The first-order condition for the choice of $\alpha_{1, t}$ can be written in the following form

$$
E_{t}\left[u^{\prime}\left(C_{t+1}\right) r_{1, t+1}\right]=E_{t}\left[u^{\prime}\left(C_{t+1}\right) r_{2, t+1}\right]
$$


Foreign agents face a similar portfolio allocation problem with a budget constraint given by

$$
W_{t}^{*}=\alpha_{1, t-1}^{*} r_{x, t}+r_{2, t} W_{t-1}^{*}+Y_{t}^{*}-C_{t}^{*}
$$

where an asterisk indicates foreign variables. In equilibrium it follows that $W_{t}^{*}=-W_{t}$. Foreign agents have preferences similar to (1) so the first-order condition for foreign agents' choice of $\alpha_{1, t}^{*}$ is

$$
E_{t}\left[u^{\prime}\left(C_{t+1}^{*}\right) r_{1, t+1}\right]=E_{t}\left[u^{\prime}\left(C_{t+1}^{*}\right) r_{2, t+1}\right]
$$

Assets are assumed to be in zero net supply, so market clearing in asset markets implies

$$
\alpha_{1, t-1}+\alpha_{1, t-1}^{*}=0, \quad \alpha_{2, t-1}+\alpha_{2, t-1}^{*}=0
$$

To simplify notation, in what follows we will drop the subscript from $\alpha_{1, t}$ and simply refer to $\alpha_{t}$. It should be understood, therefore, that $\alpha_{1, t}=-\alpha_{1, t-1}^{*}=\alpha_{t}, \alpha_{2, t}=W_{t}-\alpha_{t}$ and $\alpha_{2, t}^{*}=W_{t}^{*}+\alpha_{t}$.

Endowments are the sum of two components, so that

$$
Y_{t}=Y_{K, t}+Y_{L, t}, \quad Y_{t}^{*}=Y_{K, t}^{*}+Y_{L, t}^{*}
$$

where $Y_{K, t}$ and $Y_{K, t}^{*}$ represent 'capital income' and $Y_{L, t}$ and $Y_{L, t}^{*}$ 'labour income'. The endowments are determined by the following simple stochastic processes

$$
\begin{array}{ll}
\log Y_{K, t}=\log \bar{Y}_{K}+\varepsilon_{K, t}, & \log Y_{L, t}=\log \bar{Y}_{L}+\varepsilon_{L, t} \\
\log Y_{K, t}^{*}=\log \bar{Y}_{K}+\varepsilon_{K, t}^{*}, & \log Y_{L, t}^{*}=\log \bar{Y}_{L}+\varepsilon_{L, t}^{*}
\end{array}
$$

where $\varepsilon_{K, t}, \varepsilon_{L, t}, \varepsilon_{K, t}^{*}$ and $\varepsilon_{L, t}^{*}$ are zero-mean i.i.d. shocks which are symmetrically distributed over the interval $[-\epsilon, \epsilon]$ with $\operatorname{Var}\left[\varepsilon_{K}\right]=\operatorname{Var}\left[\varepsilon_{K}^{*}\right]=\sigma_{K}^{2}, \operatorname{Var}\left[\varepsilon_{L}\right]=\operatorname{Var}\left[\varepsilon_{L}^{*}\right]=\sigma_{L}^{2}$. We assume $\operatorname{Cov}\left[\varepsilon_{K}, \varepsilon_{K}^{*}\right]=\operatorname{Cov}\left[\varepsilon_{L}, \varepsilon_{L}^{*}\right]=0$ and $\operatorname{Cov}\left[\varepsilon_{K}, \varepsilon_{L}\right]=\operatorname{Cov}\left[\varepsilon_{K}^{*}, \varepsilon_{L}^{*}\right]=\sigma_{K L}$.

The two assets are assumed to be one-period equity claims on the home and foreign capital income. ${ }^{5}$ The real payoff to a unit of the home equity in period $t$ is defined to be

\footnotetext{
${ }^{5}$ Notice that we are assuming that, by default, all capital in a country is owned by the residents of that country. This allows us to treat equity claims to capital income as inside assets, i.e. assets in zero net supply. This is purely an accounting convention. Our solution method works equally in the alternative approach, where capital is not included in the definition of $Y$ and $Y^{*}$ and equity is treated as an outside asset which is in positive net supply. The present approach makes our derivations easier however.
} 
$Y_{K, t}$ and the real price of a unit of home equity is denoted $Z_{E, t-1}$. Thus the gross real rate of return on home equity is

$$
r_{1, t}=Y_{K, t} / Z_{E, t-1}
$$

Likewise the gross real return on foreign equity is

$$
r_{2, t}=Y_{K, t}^{*} / Z_{E, t-1}^{*}
$$

where $Z_{E, t-1}^{*}$ is the price of the foreign equity.

The first-order conditions for home and foreign consumption are

$$
C_{t}^{-\rho}=\beta E_{t}\left[C_{t+1}^{-\rho} r_{2, t+1}\right], \quad C_{t}^{*-\rho}=\beta E_{t}\left[C_{t+1}^{*-\rho} r_{2, t+1}\right]
$$

Finally, equilibrium consumption plans must satisfy the resource constraint

$$
C_{t}+C_{t}^{*}=Y_{t}+Y_{t}^{*}
$$

\subsection{Zero-order and first-order components}

Despite the extreme simplicity of this model, it is only in special cases that an exact solution can be found, e.g. when there is no labour income (in which case trade in equities supports the perfect risk-sharing equilibrium). ${ }^{6}$ The model is also not amenable to standard first-order approximation techniques, so standard linearisation approaches to DSGE models can not provide even an approximate solution to the general case. Our method, nevertheless, does yield an approximate solution to the general case. Before describing the method, it is useful to show why standard solution techniques do not work for this model, and to demonstrate how our method offers a way around the problems.

First, we define some terms relating to the true and approximate portfolio solutions. Notice that agents make their portfolio decisions at the end of each period and are free to re-arrange their portfolios each period. In a recursive equilibrium, therefore, the equilibrium asset allocation will be some function of the state of the system in each period -

\footnotetext{
${ }^{6}$ If there is no labour income then equities can be used to trade all income risk. It is easy to show that the equilibrium portfolio is for home and foreign agents to hold portfolios equally split between home and foreign equity. This implies perfect consumption risk sharing. This is a useful benchmark for comparison with the solution yielded by our method.
} 
which is summarised by the state variables. We therefore postulate that the true portfolio (i.e. the equilibrium portfolio in the non-approximated model) is a function of state variables. In the model defined above there is only one state variable, $W$ - so we postulate $\alpha_{t}=\alpha\left(W_{t}\right){ }^{7}$

Now consider a first-order Taylor-series expansion of $\alpha\left(W_{t}\right)$ around the point $W=\bar{W}$

$$
\alpha\left(W_{t}\right) \simeq \alpha(\bar{W})+\alpha^{\prime}(\bar{W})\left(W_{t}-\bar{W}\right)
$$

This approximation contains two terms: $\alpha(\bar{W})$, which is the zero-order component (i.e. $\alpha$ at the point of approximation) and $\alpha^{\prime}(\bar{W})\left(W_{t}-\bar{W}\right)$, which is the first-order component (assuming $\left(W_{t}-\bar{W}\right)$ is evaluated up to first-order accuracy). Notice that, by definition, the zero-order component of $\alpha$ is non-time varying. The approximate dynamics of the portfolio are captured by the first-order component.

When analysing a DSGE model up to first-order accuracy the standard solution approach is to use the non-stochastic steady-state of the model as the approximation point, (i.e. the zero-order component of each variable) and to use a first-order approximation of the model's equations to solve for the first-order component of each variable. Neither of these steps can be used in the above model. It is very simple to see why. In the non-stochastic equilibrium equations (4) and (6) imply

$$
r_{1, t+1}=r_{2, t+1}
$$

i.e. both assets pay the same rate of return. This implies that, for given $W$, all portfolio allocations pay the same return, so any value for $\alpha$ is consistent with equilibrium. Thus the non-stochastic steady state does not tie down a unique portfolio allocation.

A similar problem arises in a first-order approximation of the model. First-order approximation of equations (4) and (6) imply

$$
E_{t}\left[r_{1, t+1}\right]=E_{t}\left[r_{2, t+1}\right]
$$

i.e. both assets have the same expected rate of return. Again, any value of $\alpha$ is consistent with equilibrium.

\footnotetext{
${ }^{7}$ Optimal portfolio allocation will of course depend on the properties of asset returns generated by the model. In equilibrium, however, the stochastic properties of asset returns will also be a function of state variables, so the impact of asset returns on portfolio allocation is implicit in the function $\alpha\left(W_{t}\right)$.
} 
So neither the non-stochastic steady state nor a first-order approximation of the model provide enough equations to tie down the zero or first-order components of $\alpha$. The basic problem is easy to understand in economic terms. Assets in this model are only distinguishable in terms of their risk characteristics and neither the non-stochastic steady state nor a first-order approximation capture the different risk characteristics of assets. In the case of the non-stochastic steady state there is, by definition, no risk, while in a first-order approximation there is certainty equivalence.

This statement of the problem immediately suggests a solution. It is clear that the risk characteristics of assets only show up in the second-moments of model variables, and it is only by considering higher-order approximations of the model that the effects of second-moments can be captured. This fundamental insight has existed in the literature for many years. It was first formalised by Samuelson (1970), who established that, in order to derive the zero-order component of the portfolio, it is necessary to approximate the portfolio problem up to the second order. Our solution approach follows this principle. We show that a second-order approximation of the portfolio optimality conditions provides a condition which makes it possible to tie down the zero-order component of $\alpha$. The second-order approximation captures the impact of the portfolio on the correlation between portfolio returns and the marginal utility of consumption. It therefore captures differences between assets in their ability to hedge consumption risk and thus ties down an optimal portfolio allocation. In this paper we show in detail how to use second-order approximations of the portfolio optimality conditions to solve for the zero-order component of $\alpha .^{8}$

Having established this starting point, it is relatively straightforward to extend the procedure to higher-order components on $\alpha$. Samuelson (1970) in fact states a general principle that, in order to derive the $N$ th-order component of the portfolio, it is necessary to approximate the portfolio problem up to order $N+2$. In section 4 we briefly outline how, by following this principle, the solution for the first-order component of $\alpha$ can be derived from third-order approximations of the portfolio optimality conditions. The full details of the solution procedure for the first-order component are given in a companion paper, Devereux and Sutherland (2007).

\footnotetext{
${ }^{8}$ Note that Samuelson approached the problem by approximating the agent's utility function, while we take approximations of agents' first-order conditions. It is possible to show that the two approaches produce identical results.
} 
While Samuelson (1970) was the first to show how solutions for the zero and higherorder components of the portfolio may be derived, more recently Judd and Guu (2001) have demonstrated an alternative solution approach which sheds further light on the nature of the zero-order portfolio. They show how the problem of portfolio indeterminacy in the non-stochastic steady state can be overcome by using a Bifurcation theorem in conjunction with the Implicit Function Theorem. Their approach shows that the zero-order portfolio is a bifurcation point in the set of non-stochastic equilibria. Like Samuelson (1970), our solution approach relies on second-order approximations of the model to identify the zero-order component, but the underlying theory described by Judd and Guu (2001) is also applicable to our equilibrium solution. In particular, the zero-order portfolio derived using our technique corresponds to the solution that emerges from the Judd and Guu approach. Our solution can therefore be rationalised in the same way, i.e. it is a bifurcation point in the set of non-stochastic equilibria. ${ }^{9}$

The general underlying principles of the solution we derive are thus well established. The main contribution of this paper is to provide a solution approach which can easily be applied to DSGE models. ${ }^{10}$ We now demonstrate this by solving for the zero-order component of $\alpha$ in the simple two-asset endowment model described above.

\subsection{Solving for the zero-order portfolio}

In what follows, a bar over a variable indicates its value at the approximation point (i.e. the zero-order component) and a hat indicates the log-deviation from the approximation point (except in the case of $\hat{\alpha}, \hat{W}$ and $\hat{r}_{x}$, which are defined below). Notice that the non-stochastic steady state, while failing to tie down $\alpha$, still provides solutions for output, consumption and rates of return. We therefore use the non-stochastic steady state of the

\footnotetext{
${ }^{9}$ As already explained, in a non-stochastic world all portfolio allocations are equivalent and can be regarded as valid equilibria. A stochastic world on the other hand (assuming independent asset returns and suitable regularity conditions on preferences) has a unique equilibrium portfolio allocation. If one considers the limit of a sequence of stochastic worlds, with diminishing noise, the equilibrium portfolio tends towards a limit which correspond to one of the many equilibria in the non-stochastic world. This limiting portfolio is the bifurcation point described by Judd and Guu (2001), i.e. it is the point in the set of non-stochastic equilibria which intersects with the sequence of stochastic equilibria.

${ }^{10}$ Note that both Samuelson (1970) and Judd and Guu (2001) demonstrate their results using static partial equilibrium models of portfolio allocation.
} 
model as the approximation point for all variables except $\alpha$. In particular we use the symmetric non-stochastic steady state, where $\bar{W}=0$. It follows from equations (4) and (6) that $\bar{r}_{1}=\bar{r}_{2}=1 / \beta$ and thus $\bar{r}_{x}=0$. Equations (3) and (5) therefore imply that $\bar{Y}=\bar{Y}^{*}=\bar{C}=\bar{C}^{*}$. Since $\bar{W}=0$, it also follows that $\bar{\alpha}_{2}=-\bar{\alpha}_{1}=-\bar{\alpha}_{2}^{*}=\bar{\alpha}_{1}^{*}=-\bar{\alpha}$.

As argued above, solving for the zero-order component of $\alpha$ requires a second-order expansion of the portfolio problem. So we start by taking a second-order approximation of the home-country portfolio first-order condition, (4), to yield

$$
E_{t}\left[\hat{r}_{x, t+1}+\frac{1}{2}\left(\hat{r}_{1, t+1}^{2}-\hat{r}_{2, t+1}^{2}\right)-\rho \hat{C}_{t+1} \hat{r}_{x, t+1}\right]=O\left(\epsilon^{3}\right)
$$

where $\hat{r}_{x, t+1}=\hat{r}_{1, t+1}-\hat{r}_{2, t+1}$ and $O\left(\epsilon^{3}\right)$ is a residual which contains all terms of order higher than two. Applying a similar procedure to the foreign first-order condition, (6), yields

$$
E_{t}\left[\hat{r}_{x, t+1}+\frac{1}{2}\left(\hat{r}_{1, t+1}^{2}-\hat{r}_{2, t+1}^{2}\right)-\rho \hat{C}_{t+1}^{*} \hat{r}_{x, t+1}\right]=O\left(\epsilon^{3}\right)
$$

These expression can now be combined to show that, in equilibrium, the following equations must hold

$$
E_{t}\left[\left(\hat{C}_{t+1}-\hat{C}_{t+1}^{*}\right) \hat{r}_{x, t+1}\right]=0+O\left(\epsilon^{3}\right)
$$

and

$$
E_{t}\left[\hat{r}_{x, t+1}\right]=-\frac{1}{2} E_{t}\left[\hat{r}_{1, t+1}^{2}-\hat{r}_{2, t+1}^{2}\right]+\rho \frac{1}{2} E_{t}\left[\left(\hat{C}_{t+1}+\hat{C}_{t+1}^{*}\right) \hat{r}_{x, t+1}\right]+O\left(\epsilon^{3}\right)
$$

These two equations express the portfolio optimality conditions in a form which is particularly convenient for deriving equilibrium portfolio holdings and excess returns. Equation (14) provides an equation which must be satisfied by equilibrium portfolio holdings. And equation (15) shows the corresponding set of equilibrium expected excess returns.

We will now show that equation (14) provides a sufficient condition to tie down the zero-order component of $\alpha$. In order to do this we first state two important properties of the approximated model.

Property 1 In order to evaluate the left hand side of equation (14) it is sufficient to derive expressions for the first-order accurate behaviour of consumption and excess returns. This is because the only terms that appear in equation (14) are products, and second-order accurate solutions for products can be obtained from first-order accurate solutions for individual variables. 
Property 2 The only aspect of the portfolio decision that affects the first-order accurate behaviour of consumption and excess returns is $\bar{\alpha}$, i.e. the zero-order component of the $\alpha$. The first-order component, i.e. the deviation of $\alpha$ from the approximation point, does not affect the first-order behaviour of consumption and excess returns. To see why this is true notice that portfolio decisions only enter the model via the portfolio excess return, i.e. via the term $\alpha_{1, t-1} r_{x, t}$ in the budget constraints. A first-order expansion of this term is $\bar{\alpha} \hat{r}_{x, t}+\bar{r}_{x} \hat{\alpha}_{1, t-1}$. But $\bar{r}_{x}=0$ so only $\bar{\alpha} \hat{r}_{x, t}$ remains.

It is now straightforward to show that equation (14) provides a condition which ties down $\bar{\alpha}$. Property 2 tells us that it is possible to evaluate the first-order behaviour of $\left(\hat{C}_{t+1}-\hat{C}_{t+1}^{*}\right)$ and $\hat{r}_{x, t+1}$ conditional on a given value of $\bar{\alpha}$. Property 1 tells us that $E_{t}\left[\left(\hat{C}_{t+1}-\hat{C}_{t+1}^{*}\right) \hat{r}_{x, t+1}\right]$ can therefore also be evaluated conditional on a given value of $\bar{\alpha}$. Equation (14) tells us that a solution for $\bar{\alpha}$ is one which implies $E_{t}\left[\left(\hat{C}_{t+1}-\hat{C}_{t+1}^{*}\right) \hat{r}_{x, t+1}\right]=$ 0 .

In order to derive this solution for $\bar{\alpha}$ it is first necessary to solve for the first-order accurate behaviour of $\left(\hat{C}_{t+1}-\hat{C}_{t+1}^{*}\right)$ and $\hat{r}_{x, t+1}$ conditional on a given value of $\bar{\alpha}$. The first-order accurate behaviour of $\hat{r}_{x, t+1}$ is particularly simple in this model. First-order approximations of (8) and (9) imply

$$
\hat{r}_{x, t+1}=\hat{Y}_{K, t+1}-\hat{Y}_{K, t+1}^{*}-\left(\hat{Z}_{E, t}-\hat{Z}_{E, t}^{*}\right)+O\left(\epsilon^{2}\right)
$$

where $O\left(\epsilon^{2}\right)$ is a residual which contains all terms of order higher than one, so

$$
E_{t}\left[\hat{r}_{x, t+1}\right]=E_{t}\left[\hat{Y}_{K, t+1}\right]-E_{t}\left[\hat{Y}_{K, t+1}^{*}\right]-\left(\hat{Z}_{E, t}-\hat{Z}_{E, t}^{*}\right)+O\left(\epsilon^{2}\right)
$$

Notice that (15) implies that, up to a first-order approximation, $E_{t}\left[\hat{r}_{x, t+1}\right]=0$ so

$$
\left(\hat{Z}_{E, t}-\hat{Z}_{E, t}^{*}\right)=E_{t}\left[\hat{Y}_{K, t+1}\right]-E_{t}\left[\hat{Y}_{K, t+1}^{*}\right]+O\left(\epsilon^{2}\right)
$$

and thus, since $Y_{K}$ and $Y_{K}^{*}$ are i.i.d., $\hat{r}_{x, t+1}$ is given by ${ }^{11}$

$$
\hat{r}_{x, t+1}=\hat{Y}_{K, t+1}-\hat{Y}_{K, t+1}^{*}+O\left(\epsilon^{2}\right)
$$

\footnotetext{
${ }^{11}$ Notice from this derivation that, in this model, $r_{x}$ is completely independent from $\alpha$. This makes the application of our solution process particularly simple. In the next section we will show that our method can easily be applied to more general models where $\alpha$ may have a direct or indirect impact on $r_{x}$.
} 
The first-order accurate solution for $\left(\hat{C}_{t+1}-\hat{C}_{t+1}^{*}\right)$ is also straightforward to derive. A first-order approximation of the home and foreign budget constraints implies

$$
\begin{aligned}
\hat{W}_{t+1} & =\frac{1}{\beta} \hat{W}_{t}+\hat{Y}_{t+1}-\hat{C}_{t+1}+\tilde{\alpha} \hat{r}_{x, t+1}+O\left(\epsilon^{2}\right) \\
-\hat{W}_{t+1} & =-\frac{1}{\beta} \hat{W}_{t}+\hat{Y}_{t+1}^{*}-\hat{C}_{t+1}^{*}-\tilde{\alpha} \hat{r}_{x, t+1}+O\left(\epsilon^{2}\right)
\end{aligned}
$$

where $\hat{W}_{t}=\left(W_{t}-\bar{W}\right) / \bar{C}$ and $\tilde{\alpha}=\bar{\alpha} /(\beta \bar{Y})$. Combining (17) and (18) with (16) and an appropriate transversality condition implies

$$
\begin{aligned}
\sum_{i=0}^{\infty} \beta^{i} E_{t+1}\left(\hat{C}_{t+1+i}-\hat{C}_{t+1+i}^{*}\right)= & \frac{2}{\beta} \hat{W}_{t}+\left(\hat{Y}_{t+1}-\hat{Y}_{t+1}^{*}\right) \\
& +2 \tilde{\alpha}\left(\hat{Y}_{K, t+1}-\hat{Y}_{K, t+1}^{*}\right)+O\left(\epsilon^{2}\right)
\end{aligned}
$$

where use has been made of he fact that $E_{t+1}\left[\hat{Y}_{t+1+i}\right]=E_{t+1}\left[\hat{Y}_{t+1+i}^{*}\right]=E_{t+1}\left[\hat{Y}_{K, t+1+i}\right]=$ $E_{t+1}\left[\hat{Y}_{K, t+1+i}^{*}\right]=0$ for all $i>0$.

The first-order conditions for consumption, equations (10), imply

$$
E_{t+1}\left[\hat{C}_{t+1+i}-\hat{C}_{t+1+i}^{*}\right]=\hat{C}_{t+1}-\hat{C}_{t+1}^{*}+O\left(\epsilon^{2}\right) \text { for all } i>0
$$

so $\left(\hat{C}_{t+1}-\hat{C}_{t+1}^{*}\right)$ is given by

$$
\begin{aligned}
\hat{C}_{t+1}-\hat{C}_{t+1}^{*}= & \frac{2(1-\beta)}{\beta} \hat{W}_{t}+(1-\beta)\left(\hat{Y}_{t+1}-\hat{Y}_{t+1}^{*}\right) \\
& +2(1-\beta) \tilde{\alpha}\left(\hat{Y}_{K, t+1}-\hat{Y}_{K, t+1}^{*}\right)+O\left(\epsilon^{2}\right)
\end{aligned}
$$

Equations (16) and (21) show the first-order accurate behaviour of $\left(\hat{C}_{t+1}-\hat{C}_{t+1}^{*}\right)$ and $\hat{r}_{x, t+1}$ conditional on a given value of $\bar{\alpha}$. Combining these expression yields

$$
\begin{gathered}
E_{t}\left[\left(\hat{C}_{t+1}-\hat{C}_{t+1}^{*}\right) \hat{r}_{x, t+1}\right]= \\
(1-\beta) E_{t}\left[\left(\left(\hat{Y}_{t+1}-\hat{Y}_{t+1}^{*}\right)+2 \tilde{\alpha}\left(\hat{Y}_{K, t+1}-\hat{Y}_{K, t+1}^{*}\right)\right)\left(\hat{Y}_{K, t+1}-\hat{Y}_{K, t+1}^{*}\right)\right]+O\left(\epsilon^{3}\right)
\end{gathered}
$$

It follows from (14) and (22) that the solution for $\tilde{\alpha}$ is

$$
\tilde{\alpha}=-\frac{1}{2} \frac{E_{t}\left[\left(\hat{Y}_{t+1}-\hat{Y}_{t+1}^{*}\right)\left(\hat{Y}_{K, t+1}-\hat{Y}_{K, t+1}^{*}\right)\right]}{E_{t}\left[\left(\hat{Y}_{K, t+1}-\hat{Y}_{K, t+1}^{*}\right)^{2}\right]}+O(\epsilon)
$$

or

$$
\tilde{\alpha}=-\frac{\delta+(1-\delta) \sigma_{K L} / \sigma_{K}^{2}}{2}+O(\epsilon)
$$


where $\delta=\bar{Y}_{K} /\left(\bar{Y}_{K}+\bar{Y}_{L}\right)=\bar{Y}_{K} / \bar{Y}$. Notice that the residual in this expression is a first-order term. The solution for $\bar{\alpha}$ is then given by $\bar{\alpha}=\tilde{\alpha} \beta \bar{Y}$.

To provide an economic interpretation of our solution it is helpful to re-express (24) in terms of the proportion of home equity held by home residents. The total value of home equity is $\beta \bar{Y}_{K}$, so the proportion held by home residents is given by

$$
\frac{\beta \bar{Y}_{K}+\bar{\alpha}}{\beta \bar{Y}_{K}}=\frac{1+(1-\delta) \sigma_{K L} / \delta \sigma_{K}^{2}}{2}
$$

The most obvious benchmark against which to compare (25) is the case where there is no labour income, i.e. where $\delta=1$ and $\sigma_{L}^{2}=0$. In this case there is a known exact solution to the model where home and foreign agents hold a balanced portfolio of home and foreign equities. It is easy to see from (25) that our solution yields exactly this outcome. i.e. home agents hold exactly half of home equity (and by implication half of foreign equity). It is also easy to check from (21) that the equilibrium portfolio yields full consumption risk sharing. More generally, in cases where this is labour income risk, i.e. $0<\delta<1$ and $\sigma_{L}^{2}>0$, there is no exact solution to the model, but our zero-order solution provides an approximate solution. Equation (25) shows that if $\sigma_{K L}=0$ (i.e. labour and capital income are uncorrelated) agents continue to hold a balanced portfolio of home and foreign equity, but equation (21) shows that full consumption risk sharing is not achieved in this case. The equilibrium portfolio deviates from an equal balance of home and foreign equity when there is some correlation between capital and labour income. For instance, when there is a negative correlation, i.e. $\sigma_{K L}<0$, there will be home bias in equity holdings (i.e. home agents will hold more then half of home equity and foreign agents will hold more than half of foreign equity). ${ }^{12}$

Before showing how the solution procedure can be applied to a more general model, we use (24) to address a number of potentially puzzling issues. First, notice that despite the presence of time subscripts, all the terms in (23), including the conditional secondmoments, are constant. So our solution for $\bar{\alpha}$ is non-time-varying (which is consistent with our definition of the zero-order component). At first sight it may seem contradictory that portfolio allocations are non-time varying while net wealth, in the form of $\hat{W}_{t}$, is time varying. But this is to confuse orders of approximation. $\bar{\alpha}$ is the zero-order component of the portfolio, and should be compared to the zero-order component of net wealth, $\bar{W}$,

\footnotetext{
${ }^{12}$ Conversely, when $\sigma_{K L}>0$, we have a bias against home assets, as in Baxter and Jermann (1997).
} 
which, like $\bar{\alpha}$, is non-time varying. $\hat{W}_{t}$ on the other hand, is the first-order component of net wealth, and this should be compared to the first-order component of portfolios, $\hat{\alpha}_{t}$. Both $\hat{W}_{t}$ and $\hat{\alpha}_{t}$ are time varying. But notice, by Property 2 it is possible to solve for the dynamics of $\hat{W}$ without having to know the behaviour of $\hat{\alpha}$. As explained above, having solved for $\bar{\alpha}$ it is possible to solve for $\hat{\alpha}_{t}$ by analysing a third-order approximation of the portfolio problem. This is discussed below in Section 4 .

A more general implication of Property 2, which is worth emphasising, is that it is not necessary to solve for the first-order behaviour of $\hat{\alpha}$ in order to solve for the firstorder behaviour of other variables in the model. It is therefore possible to analyse the implications of the above model for the first-order behaviour of all variables other than $\alpha$ without having to solve for $\hat{\alpha}$.

The logic presented above implies that the zero-order component of the portfolio, $\bar{\alpha}$, is analogous to the zero-order component of the other variables in the model. At first sight this may also seem contradictory, since the zero-order components of other variables are derived from the non-stochastic steady state, while our solution for $\bar{\alpha}$ is derived from an explicitly stochastic analysis. The way to resolve this apparent contradiction is to interpret $\bar{\alpha}$ as the equilibrium for portfolio holdings in a world with an arbitrarily small amount of stochastic noise, i.e. the equilibrium in a 'near-non-stochastic' world. If one considers the limit of a sequence of stochastic worlds, with diminishing noise, the equilibrium portfolio tends towards a limit which correspond to one of the many portfolio equilibria in the non-stochastic world. This limiting portfolio is a bifurcation point described by Judd and Guu (2001), i.e. it is the point in the set of non-stochastic equilibria which intersects with the sequence of stochastic equilibria. Our solution for $\bar{\alpha}$ corresponds to the portfolio allocation at this bifurcation point. ${ }^{13}$

Finally, we note a technical issue that arises regarding the point of approximation

\footnotetext{
${ }^{13}$ Suppose that the covariance matrix of the innovations is given by $\Sigma=\zeta \Sigma_{0}$ where $\zeta>0$ is a scalar and $\Sigma_{0}$ is a valid covariance matrix. Notice that the solution for $\tilde{\alpha}$ given in (24) is independent of $\zeta$. So the value of $\tilde{\alpha}$ given by (24) (and therefore the value of $\bar{\alpha}$ ) is equivalent to the value that would arise in the case of an arbitrarily small, but non-zero, value of $\zeta$ - i.e. the value of $\tilde{\alpha}$ that would arise in a world which is arbitrarily close to a non-stochastic world. Furthermore, notice that as $\epsilon$ tends to zero (which is equivalent to $\zeta$ tending to zero) the size of the residual in (24) tends to zero. So, as the amount of noise tends to zero, the value of $\tilde{\alpha}$ becomes arbitrarily close to the true value of portfolio holdings in the non-approximated model. Our solution for $\bar{\alpha}$ can therefore be thought of as the true portfolio equilibrium in a world which is arbitrarily close to the non-stochastic equilibrium.
} 
of $W_{t}$. In the example given above, there is a unit root in the dynamics of net foreign assets at the level of first-order approximation. This means that we would not be able to compute unconditional second moments from the model. But this has no bearing on the portfolio solution. Equilibrium portfolios depend only on conditional second moments, which are well defined. The unit root property could easily be eliminated using any of the approaches discussed in Schmitt-Grohe and Uribe (2003), and it should be clear from the above presentation that our approach works equally well in this case. We chose to use the model here however, because it gives very simple and intuitive expressions for optimal portfolios.

\section{Generalising to an n-Asset Model}

\subsection{The Model}

We now show how the solution method can be extended to a much more general model with many assets. The model we now describe is general enough to encompass the range of structures that are widely used in the recent open economy macro literature. However, only those parts of the model directly necessary for understanding the portfolio selection problem need to be explicitly described. Other components of the model, such as the labour supply decisions of households and the production and pricing decisions of firms, are not directly relevant to the portfolio allocation problem, so these parts of the model are suppressed. The solution approach is consistent with a wide range of specifications for labour supply, pricing and production. Thus, the non-portfolio parts of the model may be characterised by endogenous or exogenous employment, sticky or flexible prices and wages, local currency pricing or producer currency pricing, perfect competition or imperfect competition, etc.

We continue to assume that the world consists of two countries. The home country is assumed to produce a good (or a bundle of goods) with aggregate quantity denoted $Y_{H}$ (which can be endogenous) and aggregate price $P_{H}$. Similarly the foreign country produces quantity $Y_{F}$ of a (potentially differentiated) foreign good (or bundle of goods) at price $P_{F}^{*}$. In what follows foreign currency prices are denoted with an asterisk. 
Agents in the home country now have a utility function of the form

$$
U_{t}=E_{t} \sum_{\tau=t}^{\infty} \beta^{\tau-t}\left[u\left(C_{\tau}\right)+v(.)\right]
$$

where $C$ is a bundle of the home and foreign goods and $u($.$) is a twice continuously differ-$ entiable period utility function. The function $v($.$) captures those parts of the preference$ function which are not relevant for the portfolio problem. ${ }^{14}$ The aggregate consumer price index for home agents is denoted $P$.

There are $n$ assets and a vector of $n$ returns (for holdings of assets from period $t-1$ to $t$ ) given by

$$
r_{t}^{\prime}=\left[\begin{array}{llll}
r_{1, t} & r_{2, t} & \ldots & r_{n, t}
\end{array}\right]
$$

Asset payoffs and asset prices are measured in terms of the aggregate consumption good of the home economy (i.e. in units of $C$ ). Returns are defined to be the sum of the payoff of the asset and capital gains relative to the asset price. As before, it is assumed that the vector of available assets is exogenous and predefined.

The budget constraint for home agents is given by

$$
\sum \alpha_{i, t}=\sum r_{i, t} \alpha_{i, t-1}+Y_{t}-C_{t}
$$

where $\left[\alpha_{1, t-1}, \alpha_{2, t-1} \ldots \alpha_{n, t-1}\right]$ are the holdings of the $n$ assets purchased at the end of period $t-1$ for holding into period $t . Y$ is the total disposable income of home agents expressed in terms of the home consumption good. Thus, $Y$ may be given by $Y_{H} P_{H} / P+T$ where $T$ is a fiscal transfer (or tax if negative). ${ }^{15}$

Using the following definition of net wealth (net foreign assets)

$$
W_{t}=\sum_{i} \alpha_{i, t}
$$

\footnotetext{
${ }^{14}$ For these other aspects of the preference function to be irrelevant for portfolio selection it is necessary to assume utility is additively separable in $u(C)$ and $v($.$) . Extensions to cases of non-additive separability$ (e.g. habit persistence in consumption) are straightforward, as will become more clear below. Using (26) allows us to illustrate the method with minimal notation.

${ }^{15}$ Without changing any of the results below, we could augment $Y$ to allow for convex adjustment costs in $W$ arising from having net foreign assets away from their long term mean $\bar{W}$. This would ensure a stationary distribution for $W$. Thus, the model developed in this section does not necessarily display the unit root property for $W$.
} 
the budget constraint may be re-written in the following form

$$
W_{t}=\alpha_{t-1}^{\prime} r_{x, t}+r_{n, t} W_{t-1}+Y_{t}-C_{t}
$$

where

$$
\alpha_{t-1}^{\prime}=\left[\begin{array}{llll}
\alpha_{1, t-1} & \alpha_{2, t-1} & \ldots & \alpha_{n-1, t-1}
\end{array}\right]
$$

and

$$
r_{x, t}^{\prime}=\left[\begin{array}{llll}
\left(r_{1, t}-r_{n, t}\right) & \left(r_{2, t}-r_{n, t}\right) & \ldots & \left(r_{n-1, t}-r_{n, t}\right)
\end{array}\right]=\left[\begin{array}{llll}
r_{x, 1, t} & r_{x, 2, t} & \ldots & r_{x, n-1, t}
\end{array}\right]
$$

Here the $n$th asset is used as a numeraire and $r_{x, t}$ measures the "excess returns" on the other $n-1$ assets.

There are $n-1$ first-order conditions for the choice of the elements of $\alpha_{t}$ which can be written in the following form

$$
\begin{aligned}
E_{t}\left[u^{\prime}\left(C_{t+1}\right) r_{1, t+1}\right] & =E_{t}\left[u^{\prime}\left(C_{t+1}\right) r_{n, t+1}\right] \\
E_{t}\left[u^{\prime}\left(C_{t+1}\right) r_{2, t+1}\right] & =E_{t}\left[u^{\prime}\left(C_{t+1}\right) r_{n, t+1}\right] \\
& : \\
E_{t}\left[u^{\prime}\left(C_{t+1}\right) r_{n-1, t+1}\right] & =E_{t}\left[u^{\prime}\left(C_{t+1}\right) r_{n, t+1}\right]
\end{aligned}
$$

Foreign-country agents face a similar portfolio allocation problem with a budget constraint given by

$$
\frac{1}{Q_{t}} W_{t}^{*}=\frac{1}{Q_{t}}\left[\alpha_{t-1}^{* \prime} r_{x, t}+r_{n, t} W_{t-1}^{*}\right]+Y_{t}^{*}-C_{t}^{*}
$$

where $Q_{t}=P_{t}^{*} S_{t} / P_{t}$ is the real exchange rate. The real exchange rate enters this budget constraint because $Y^{*}$ and $C^{*}$ are measured in terms of the foreign aggregate consumption good (which may differ from the home consumption good) while asset holdings and rates of return are defined in terms of the home consumption good.

Foreign agents are assumed to have preferences similar to (26) so the first-order conditions for foreign-country agents' choice of $\alpha_{t}^{*}$ are

$$
\begin{aligned}
E_{t}\left[Q_{t+1}^{-1} u^{\prime}\left(C_{t+1}^{*}\right) r_{1, t+1}\right] & =E_{t}\left[Q_{t+1}^{-1} u^{\prime}\left(C_{t+1}^{*}\right) r_{n, t+1}\right] \\
E_{t}\left[Q_{t+1}^{-1} u^{\prime}\left(C_{t+1}^{*}\right) r_{2, t+1}\right] & =E_{t}\left[Q_{t+1}^{-1} u^{\prime}\left(C_{t+1}^{*}\right) r_{n, t+1}\right] \\
& : \\
E_{t}\left[Q_{t+1}^{-1} u^{\prime}\left(C_{t+1}^{*}\right) r_{n-1, t+1}\right] & =E_{t}\left[Q_{t+1}^{-1} u^{\prime}\left(C_{t+1}^{*}\right) r_{n, t+1}\right]
\end{aligned}
$$

The two sets of first-order conditions, (30) and (32), and the market clearing condition $\alpha_{t}=-\alpha_{t}^{*}$, provide $3(n-1)$ equations which determine the elements of $\alpha_{t}, \alpha_{t}^{*}$ and $E_{t}\left[r_{x, t+1}\right]$. 
Clearly, in any particular general equilibrium model, there will be a set of first-order conditions relating to intertemporal choice of consumption, labour supply, etc., for the home and foreign consumers, and a set of first-order conditions for price setting and factor demands for home and foreign producers. Taken as a whole, and combined with an appropriate set of equilibrium conditions for goods and factor markets, this full set of equations will define the general equilibrium of the model. As already explained, the details of these non-portfolio parts of the model are not necessary for the exposition of the solution method, so they are not shown explicitly. In what follows these omitted equations are simply referred to as the "non-portfolio equations" of the model.

The non-portfolio equations of the model will normally include some exogenous forcing variables. In the typical macroeconomic model these take the form of AR1 processes which are driven by zero-mean i.i.d. innovations. We assume that there are $m$ such disturbances, summarised in a vector, $x$, which is determined by the following process

$$
x_{t}=N x_{t-1}+\varepsilon_{t}
$$

where $\varepsilon$ is a vector of zero-mean i.i.d. innovations with covariance matrix $\Sigma$. It is assumed that the innovations are symmetrically distributed over the interval $[-\epsilon, \epsilon]{ }^{16}$

\subsection{Solving for the zero-order portfolio}

Again we use the symmetric non-stochastic steady state of the model as the approximation point for non-portfolio variables. Thus $\bar{W}=0, \bar{Y}=\bar{Y}^{*}=\bar{C}=\bar{C}^{*}$ and $\bar{r}_{1}=\bar{r}_{2} \ldots=\bar{r}_{n}=$ $1 / \beta$. Note again that this implies $\bar{r}_{x}=0$.

As before we proceed by taking second-order approximations of the home and foreign portfolio first-order conditions. For the home country this yields

$$
\begin{gathered}
E_{t}\left[\left(\hat{r}_{1, t+1}-\hat{r}_{n, t+1}\right)+\frac{1}{2}\left(\hat{r}_{1, t+1}^{2}-\hat{r}_{n, t+1}^{2}\right)-\rho \hat{C}_{t+1}\left(\hat{r}_{1, t+1}-\hat{r}_{n, t+1}\right)\right]=O\left(\epsilon^{3}\right) \\
E_{t}\left[\left(\hat{r}_{2, t+1}-\hat{r}_{n, t+1}\right)+\frac{1}{2}\left(\hat{r}_{2, t+1}^{2}-\hat{r}_{n, t+1}^{2}\right)-\rho \hat{C}_{t+1}\left(\hat{r}_{2, t+1}-\hat{r}_{n, t+1}\right)\right]=O\left(\epsilon^{3}\right) \\
: \\
E_{t}\left[\left(\hat{r}_{n-1, t+1}-\hat{r}_{n, t+1}\right)+\frac{1}{2}\left(\hat{r}_{n-1, t+1}^{2}-\hat{r}_{n, t+1}^{2}\right)-\rho \hat{C}_{t+1}\left(\hat{r}_{n-1, t+1}-\hat{r}_{n, t+1}\right)\right]=O\left(\epsilon^{3}\right)
\end{gathered}
$$

\footnotetext{
${ }^{16}$ Clearly there must be a link between $\Sigma$ and $\epsilon$. The value of $\epsilon$ places an upper bound on the diagonal elements of $\Sigma$. So an experiment which involves considering the effects of reducing $\epsilon$ implicitly involves reducing the magnitude of the elements of $\Sigma$.
} 
where $\rho \equiv-u^{\prime \prime}(\bar{C}) \bar{C} / u^{\prime}(\bar{C})$ (i.e. the coefficient of relative risk aversion). Re-writing (34) in vector form yields

$$
E_{t}\left[\hat{r}_{x, t+1}+\frac{1}{2} \hat{r}_{x, t+1}^{2}-\rho \hat{C}_{t+1} \hat{r}_{x, t+1}\right]=O\left(\epsilon^{3}\right)
$$

where

$$
\hat{r}_{x, t+1}^{\prime} \equiv\left[\begin{array}{llll}
\hat{r}_{1, t+1}-\hat{r}_{n, t+1} & \hat{r}_{2, t+1}-\hat{r}_{n, t+1} \quad \ldots \quad \hat{r}_{n-1, t+1}-\hat{r}_{n, t+1}
\end{array}\right]
$$

and

$$
\hat{r}_{x, t+1}^{2 \prime} \equiv\left[\begin{array}{llll}
\hat{r}_{1, t+1}^{2}-\hat{r}_{n, t+1}^{2} & \hat{r}_{2, t+1}^{2}-\hat{r}_{n, t+1}^{2} \quad \ldots \quad \hat{r}_{n-1, t+1}^{2}-\hat{r}_{n, t+1}^{2}
\end{array}\right]
$$

Applying a similar procedure to the foreign first-order conditions yields

$$
E_{t}\left[\hat{r}_{x, t+1}+\frac{1}{2} \hat{r}_{x, t+1}^{2}-\rho \hat{C}_{t+1}^{*} \hat{r}_{x, t+1}+\hat{Q}_{t+1}\right]=0+O\left(\epsilon^{3}\right)
$$

The home and foreign optimality conditions, (35) and (36), can be combined to show that, in equilibrium, the following conditions must hold

$$
E_{t}\left[\left(\hat{C}_{t+1}-\hat{C}_{t+1}^{*}-\hat{Q}_{t+1} / \rho\right) \hat{r}_{x, t+1}\right]=0+O\left(\epsilon^{3}\right)
$$

and

$$
E\left[\hat{r}_{x}\right]=-\frac{1}{2} E\left[\hat{r}_{x}^{2}\right]+\rho \frac{1}{2} E_{t}\left[\left(\hat{C}_{t+1}+\hat{C}_{t+1}^{*}+\hat{Q}_{t+1} / \rho\right) \hat{r}_{x, t+1}\right]+O\left(\epsilon^{3}\right)
$$

These equations are equivalent to (14) and (15) in the example from before. There we showed that equation (14) provided a sufficient condition to tie down the zero-order component of the portfolio allocation. We now show that equation (37) provides a sufficient condition to tie down the zero-order component of the portfolio in the general model.

Properties 1 and 2 played a central role in deriving the solution to the example above. These properties also hold for the general model, and remain central in the derivation of the solution. Clearly, Property 1 applies in the general model. The left hand side of equation (37) consists entirely of products of variables and can thus be evaluated to second-order accuracy using first-order accurate expressions for $\hat{C}-\hat{C}^{*}-\hat{Q} / \rho$ and $\hat{r}_{x}$. Likewise, Property 2 holds in the general model. Again, the portfolio allocation enters only via the excess portfolio return, $\alpha^{\prime} r_{x}$. And, just as in the simple model, $\bar{r}_{x}=0$, so the first-order approximation of the excess portfolio return is $\bar{\alpha} \hat{r}_{x}$. Thus only the zero-order component of $\alpha$ enters the first-order approximated model. 
The general outline of the solution strategy is the same as that described for the simple model. First we solve for the first-order accurate behaviour of $\hat{C}-\hat{C}^{*}-\hat{Q} / \rho$ and $\hat{r}_{x}$ in terms of $\bar{\alpha}$. Then we solve for the $\bar{\alpha}$ that ensures (37) is satisfied.

But now things are somewhat more complicated because the behaviour of $\hat{C}-\hat{C}^{*}-\hat{Q} / \rho$ and $\hat{r}_{x}$ is determined by a potentially complex set of first-order dynamic equations. Indeed, at first sight, the general model may seem too complex to be solved explicitly, and it may appear that a numerical approach is necessary to solve for the $\bar{\alpha}$. We show, however, that it is possible to derive a closed-form analytical solution for $\bar{\alpha}$ in the general model. In fact, we derive a formula for $\bar{\alpha}$ which is applicable to any model with the same general features as the one described above.

To see why it is possible to obtain a closed-form solution, it is necessary to state a further important property of the approximated model.

Property 3 To a first-order approximation, the portfolio excess return, $\bar{\alpha} \hat{r}_{x, t+1}$, is a zero mean i.i.d. random variable. This follows from equation (38), which shows that the equilibrium expected excess return contains only second-order terms. So, up to a first order approximation, $E_{t-1}\left[\hat{r}_{x, t+1}\right]$ is zero, i.e. there is no predictable element in $\hat{r}_{x, t+1}$. The first-order approximation of the portfolio excess return, $\bar{\alpha} \hat{r}_{x, t+1}$, is therefore a linear function of the i.i.d. innovations, $\varepsilon_{t+1}$, and must therefore itself be an i.i.d. random variable.

Property 3 greatly simplifies the solution process because it implies that $\bar{\alpha}$ affects the first-order behaviour of the economy in a very simple way. In particular, $\bar{\alpha}$ does not affect the eigenvalues of the first-order system. Thus, in any given period (e.g. period $t$ ) the dynamic properties of the expected path of the economy from period $t+1$ onwards are independent of $\bar{\alpha}$. The period $t$ behaviour of the economy is affected by $\bar{\alpha}$ only through its effect on the size and sign of i.i.d. innovations to wealth arising from the portfolio excess return, $\bar{\alpha} \hat{r}_{x, t}$.

The only remaining potential complication is that $\hat{r}_{x, t}$ may itself depend on period $t$ innovations to wealth (and therefore $\bar{\alpha}$ ). This complication is, however, easily overcome by breaking the solution process for $\hat{C}-\hat{C}^{*}-\hat{Q} / \rho$ and $\hat{r}_{x}$ into two stages. In the first stage we treat the portfolio excess return, $\bar{\alpha} \hat{r}_{x}$, as an exogenous i.i.d. random variable, and solve the first-order model to yield an expression for $\hat{r}_{x}$ in terms of exogenous innovations to wealth. In the second stage we use this expression to solve out for the behaviour of 
$\hat{C}-\hat{C}^{*}-\hat{Q} / \rho$ and $\hat{r}_{x}$ in terms of $\varepsilon$ (i.e. the true exogenous innovations of the model). This provides the expressions required to evaluate (37) and thus to solve for $\bar{\alpha} .^{17}$

We now apply this procedure to the general model. First note that the first-order approximation of the home budget constraint is given by

$$
\hat{W}_{t}=\frac{1}{\beta} \hat{W}_{t-1}+\hat{Y}_{t}-\hat{C}_{t}+\tilde{\alpha}^{\prime} \hat{r}_{x t}+O\left(\epsilon^{2}\right)
$$

where $\hat{W}_{t}=\left(W_{t}-\bar{W}\right) / \bar{Y}$ and $\tilde{\alpha}=\bar{\alpha} /(\beta \bar{Y})$. The solution procedure will be described in terms of deriving a solution for $\tilde{\alpha}$. The corresponding solution for $\bar{\alpha}$ is obviously given by $\bar{\alpha}=\tilde{\alpha} \beta \bar{Y}$. We now rewrite the budget constraint in the form

$$
\hat{W}_{t}=\frac{1}{\beta} \hat{W}_{t-1}+\hat{Y}_{t}-\hat{C}_{t}+\xi_{t}+O\left(\epsilon^{2}\right)
$$

where $\tilde{\alpha}^{\prime} \hat{r}_{x t}$ has been replaced by $\xi_{t}$. We temporarily treat $\xi$ as an exogenous i.i.d. variable.

The first-order approximation of the model can now be summarised in a matrix equation of the form

$$
A_{1}\left[\begin{array}{c}
s_{t+1} \\
E_{t}\left[c_{t+1}\right]
\end{array}\right]=A_{2}\left[\begin{array}{c}
s_{t} \\
c_{t}
\end{array}\right]+A_{3} x_{t}+B \xi_{t}+O\left(\epsilon^{2}\right)
$$

where $s$ is the vector of predetermined variables, $c$ is the vector of jump variables, $x$ is defined in (33) and $B$ is a column vector with unity in the row corresponding to (39) and zero in all other rows. The state-space solution to (40) can be derived using any standard solution method for linear rational expectations models. It can be written as follows

$$
\begin{aligned}
& s_{t+1}=F_{1} x_{t}+F_{2} s_{t}+F_{3} \xi_{t}+O\left(\epsilon^{2}\right) \\
& c_{t}=P_{1} x_{t}+P_{2} s_{t}+P_{3} \xi_{t}+O\left(\epsilon^{2}\right)
\end{aligned}
$$

By extracting the appropriate rows from (41) it is possible to write the following expression for the first-order accurate relationship between excess returns, $\hat{r}_{x t+1}$, and $\varepsilon_{t+1}$ and $\xi_{t+1}$

$$
\hat{r}_{x t+1}=R_{1} \xi_{t+1}+R_{2} \varepsilon_{t+1}+O\left(\epsilon^{2}\right)
$$

where the matrices $R_{1}$ and $R_{2}$ are formed from the appropriate rows of (41). Equation (42) shows how first-order accurate realised excess returns depend on exogenous i.i.d. shocks,

\footnotetext{
${ }^{17}$ Notice from equation (15) that, in the example, $r_{x}$ does not depend on $\bar{\alpha}$, so this two-step process for was not necessary.
} 
$\varepsilon_{t+1}$ and $\xi_{t+1} \cdot{ }^{18}$ In particular, it shows how $\hat{r}_{x t+1}$ depends on i.i.d. shocks to wealth. This completes the first stage in solving for the first-order behaviour of $\hat{C}-\hat{C}^{*}-\hat{Q} / \rho$ and $\hat{r}_{x}$.

Now we impose the condition that, rather than being exogenous, the innovations to wealth, $\xi_{t+1}$, are endogenously determined by excess portfolio returns via the relationship

$$
\xi_{t+1}=\tilde{\alpha}^{\prime} \hat{r}_{x t+1}
$$

where the vector of portfolio allocations, $\tilde{\alpha}$, is yet to be determined. This equation, together with (42), can be solved to yield expressions for $\xi_{t+1}$ and $\hat{r}_{x t+1}$ in terms of the exogenous innovations as follows

$$
\begin{gathered}
\xi_{t+1}=\tilde{H} \varepsilon_{t+1} \\
\hat{r}_{x t+1}=\tilde{R} \varepsilon_{t+1}+O\left(\epsilon^{2}\right)
\end{gathered}
$$

where

$$
\tilde{H}=\frac{\tilde{\alpha}^{\prime} R_{2}}{1-\tilde{\alpha}^{\prime} R_{1}}, \quad \tilde{R}=R_{1} \tilde{H}+R_{2}
$$

Equation (45), which shows how realised excess returns depend on the exogenous i.i.d. innovations of the model, provides one of the relationships necessary to evaluate the lefthand side of (37). The other relationship required is the link between $\hat{C}_{t+1}-\hat{C}_{t+1}^{*}-\hat{Q}_{t+1} / \rho$ and the vector of exogenous innovations, $\varepsilon_{t+1}$. This relationship can derived in a similar way to (45). First extract the appropriate rows from (41) to yield the following

$$
\hat{C}_{t+1}-\hat{C}_{t+1}^{*}-\hat{Q}_{t+1} / \rho=D_{1} \xi_{t+1}+D_{2} \varepsilon_{t+1}+D_{3}\left[\begin{array}{c}
x_{t} \\
s_{t+1}
\end{array}\right]+O\left(\epsilon^{2}\right)
$$

where the matrices $D_{1}, D_{2}$ and $D_{3}$ are formed from the appropriate rows of (41). After substituting for $\xi_{t+1}$ using (44) this implies

$$
\hat{C}_{t+1}-\hat{C}_{t+1}^{*}-\hat{Q}_{t+1} / \rho=\tilde{D} \varepsilon_{t+1}+D_{3}\left[\begin{array}{c}
x_{t} \\
s_{t+1}
\end{array}\right]+O\left(\epsilon^{2}\right)
$$

where

$$
\tilde{D}=D_{1} \tilde{H}+D_{2}
$$

Equations (45) and (48) are the equivalents of (16) and (21) in the example. They show the first-order accurate behaviour of $\hat{r}_{x t+1}$ and $\hat{C}_{t+1}-\hat{C}_{t+1}^{*}-\hat{Q}_{t+1} / \rho$ and they can

\footnotetext{
${ }^{18}$ Notice that, as follows from Property $3, \hat{r}_{x t+1}$ does not depend on the values of the state variables contained in $x_{t}$ or $s_{t}$.
} 
be used to evaluate the second-order accurate behaviour of the left hand side of equation (37), as follows

$$
E_{t}\left[\left(\hat{C}_{t+1}-\hat{C}_{t+1}^{*}-\hat{Q}_{t+1} / \rho\right) \hat{r}_{x, t+1}\right]=\tilde{R} \Sigma \tilde{D}^{\prime}+O\left(\epsilon^{3}\right)
$$

where $\Sigma$ is the covariance matrix of $\varepsilon{ }^{19}$ The equilibrium value of $\tilde{\alpha}$ satisfies the following equation

$$
\tilde{R} \Sigma \tilde{D}^{\prime}=0
$$

This matrix equation defines $(n-1)$ equations in the $(n-1)$ elements of $\tilde{\alpha}$.

To solve for $\tilde{\alpha}$ first substitute for $\tilde{R}$ and $\tilde{D}$ in (51) and expand to yield

$$
R_{1} \tilde{H} \Sigma \tilde{H}^{\prime} D_{1}^{\prime}+R_{2} \Sigma \tilde{H}^{\prime} D_{1}^{\prime}+R_{1} \tilde{H} \Sigma D_{2}^{\prime}+R_{2} \Sigma D_{2}^{\prime}=0+O\left(\epsilon^{3}\right)
$$

Substituting for $\tilde{H}$ and $\tilde{H}^{\prime}$ and multiplying by $\left(1-\tilde{\alpha}^{\prime} R_{1}\right)^{2}$ yields

$$
\begin{aligned}
& R_{1} \tilde{\alpha}^{\prime} R_{2} \Sigma R_{2}^{\prime} \tilde{\alpha} D_{1}^{\prime}+R_{2} \Sigma R_{2}^{\prime} \tilde{\alpha} D_{1}^{\prime}\left(1-\tilde{\alpha}^{\prime} R_{1}\right) \\
& \quad+R_{1} \tilde{\alpha}^{\prime} R_{2} \Sigma D_{2}^{\prime}\left(1-\tilde{\alpha}^{\prime} R_{1}\right)+R_{2} \Sigma D_{2}^{\prime}\left(1-\tilde{\alpha}^{\prime} R_{1}\right)^{2}=0+O\left(\epsilon^{3}\right)
\end{aligned}
$$

Note that $\tilde{\alpha}^{\prime} R_{1},\left(1-\tilde{\alpha}^{\prime} R_{1}\right)$ and $D_{1}$ are all scalars. It therefore follows that $\tilde{\alpha}^{\prime} R_{1}=R_{1}^{\prime} \tilde{\alpha}$ and $D_{1}^{\prime}=D_{1}$. Using these facts (53) simplifies to

$$
D_{1} R_{2} \Sigma R_{2}^{\prime} \tilde{\alpha}-R_{2} \Sigma D_{2}^{\prime} R_{1}^{\prime} \tilde{\alpha}+R_{2} \Sigma D_{2}^{\prime}=0+O\left(\epsilon^{3}\right)
$$

which can be solved to yield the following expression for the equilibrium $\tilde{\alpha}$

$$
\tilde{\alpha}=\left[R_{2} \Sigma D_{2}^{\prime} R_{1}^{\prime}-D_{1} R_{2} \Sigma R_{2}^{\prime}\right]^{-1} R_{2} \Sigma D_{2}^{\prime}+O(\epsilon)
$$

Notice that the residual in this expression is a first-order term. As previously noted, the solution for $\bar{\alpha}$ is simply given by $\bar{\alpha}=\tilde{\alpha} \beta \bar{Y}$.

\subsection{Summary of the procedure}

It should be emphasized that implementing this procedure requires only that the user apply (55), which needs only information from the first-order approximation of the model in order to construct the $D$ and $R$ matrices. So long as the model satisfies the general properties described above, the other details of the model, such as production, labour supply,

\footnotetext{
${ }^{19}$ Notice $D_{3}$ does not appear in this expression because, by assumption, $E_{t}\left(\varepsilon_{t+1} x_{t}\right)=E_{t}\left(\varepsilon_{t+1} s_{t+1}\right)=0$.
} 
and price setting can be varied without affecting the implementation. The derivations used to obtain (55) do not need to be repeated. In summary, the solution for equilibrium $\tilde{\alpha}$ has three steps:

1. Solve the non-portfolio equations of the model in the form of (40) to yield a solution in the form of (41).

2. Extract the appropriate rows from this solution to form $R_{1}, D_{1}, R_{2}$ and $D_{2}$.

3. Calculate $\tilde{\alpha}$ using (55).

\section{Solving for the first-order portfolio}

The analysis presented above shows how a second-order approximation of the portfolio optimality condition provides a sufficient condition to tie down the zero-order component of the portfolio, $\bar{\alpha}$. We have shown that, from Property 2, the solution for $\bar{\alpha}$ is all that is required to derive first-order accurate solutions for all other variables of a model. Thus, if the objective is to analyse the impulse responses of variables such as output or consumption (or indeed any variable other than $\alpha$ ), or if one is primarily interested in the business cycle properties of a model, then there is no need to go any further than obtaining a solution for $\bar{\alpha}$. It is likely however that the first-order dynamic behaviour of $\alpha$ will also prove to be an interesting topic of research in its own right. For instance, we might like to analyze the separate movement in different types of assets and gross portfolio positions following macro shocks. In addition, to conduct welfare analysis, we would generally need to evaluate the model up to a second-order approximation, which would require incorporating the dynamic properties of $\alpha$. We therefore now briefly outline how the solution approach can be extended to solve for the first-order component of $\alpha$.

The general principles that underlie an extension of the procedure are simply stated. In line with Samuelson (1970) it is necessary to approximate the portfolio problem up to the third order. In the context of the simple model this involves a third-order approximation of the portfolio optimality condition, as follows

$$
E_{t}\left[\begin{array}{c}
-\rho\left(\hat{C}_{t+1}-\hat{C}_{t+1}^{*}\right) \hat{r}_{x, t+1}+\frac{\rho^{2}}{2}\left(\hat{C}_{t+1}^{2}-\hat{C}_{t+1}^{* 2}\right) \hat{r}_{x, t+1} \\
-\frac{\rho}{2}\left(\hat{C}_{t+1}-\hat{C}_{t+1}^{*}\right)\left(\hat{r}_{1, t+1}^{2}-\hat{r}_{2, t+1}^{2}\right)
\end{array}\right]=0+O\left(\epsilon^{4}\right)
$$


It is now possible to show, using modified versions of Properties 1 and 2, that (56) provides a sufficient condition to tie down the first-order component of $\alpha$.

A modified version of Property 1 states that the expression on the left hand side of (56) can be evaluated up to third-order accuracy using first and second-order accurate expressions for $\hat{C}-\hat{C}^{*}, \hat{r}_{1}, \hat{r}_{1}$ and $\hat{r}_{x}$. Thus it is, at most, necessary to evaluate these variables up to second order.

A modified version of Property 2 states that only the zero and first-order components of $\alpha$ enter a second-order approximation of the model. This is simple to show by taking a second-order approximation of the portfolio excess return, $\alpha_{1, t-1} r_{x, t}$, as follows

$$
\bar{\alpha} \hat{r}_{x, t}+\bar{r}_{x} \hat{\alpha}_{1, t-1}+\frac{1}{2} \bar{\alpha}\left(\hat{r}_{1, t}^{2}-\hat{r}_{2, t}^{2}\right)+\hat{\alpha}_{t-1} \hat{r}_{x, t}+O\left(\epsilon^{3}\right)
$$

where $\hat{\alpha}_{t}=\left(\alpha_{t}-\bar{\alpha}\right)$. As before $\bar{r}_{x}=0$, so only the zero and first-order components of $\alpha$ are necessary to evaluate (57).

The general solution strategy can now be described. First, postulate that, up to firstorder accuracy, $\hat{\alpha}_{t}$ is a linear function of the state variables of the model. Thus postulate $\hat{\alpha}_{t-1}=\gamma^{\prime} z_{t}$ where $z$ is the vector of state variables and $\gamma$ is a vector of coefficients which are to be determined. The modified version of Property 2 shows that it possible to evaluate the first and second-order behaviour of $\hat{C}-\hat{C}^{*}, \hat{r}_{1}, \hat{r}_{1}$ and $\hat{r}_{x}$ conditional on a value for $\gamma$, and hence, from the modified version of Property 1 , it is possible to evaluate the left hand side of (56) conditional on $\gamma$. The equilibrium $\gamma$ is the one which ensures (56) is satisfied. ${ }^{20}$

The details of the solution procedure for $\gamma$ are presented in Devereux and Sutherland (2007), where we derive a closed-form solution which is applicable to a wide class of models.

\section{Conclusion}

Portfolio structure has become a central issue in open economy macroeconomics and international finance. Despite this, existing models and solution methods are not well-suited to analyzing portfolio choice in policy-relevant general equilibrium environments. This

\footnotetext{
${ }^{20}$ Note that the conditional third moments in (56) are time varying and depend on state variables. The fact that (56) must be satisfied for all values of state variables and in all time periods provides just enough equations to tie down all the elements of $\gamma$.
} 
paper develops a simple approximation method for portfolio choice problems in dynamic general equilibrium models. Our approach is extremely easy to implement and can be used in any of the existing models that rely on first-order approximation methods. If the researcher is primarily interested in the implications of portfolio choice for the first-order properties of macro variables (such as GDP, consumption, or the real exchange rate), either through impulse response analysis or by computing second moments so as to describe volatility and comovement, then the solution method outlined here allows a full answer to these questions. Since the overwhelming majority of the research in international finance and macroeconomics is carried out at the level of first-order approximation, the method is widely applicable. It can be used to study many empirical questions in the interface between international finance and macroeconomics. Moreover, the method allows us to study the macroeconomic determinants of optimal steady-state portfolio holdings for any asset or combination of assets, whether markets are complete or incomplete.

We note that, although the motivation and applications discussed in the paper pertain to open economy macro models, there is nothing inherent in the solution approach which restricts the application to open economies. The method applies to portfolio choice in any heterogeneous agent models dynamic general equilibrium models. This is true for both the zero-order portfolio solution, as well as the first-order solution for portfolio dynamics. Taken in combination, the methods described here offer a tractable approach to incorporating financial structure into a wide class of stochastic dynamic general equilibrium models.

\section{References}

[1] Baxter, M and U Jermann (1997) "The International Diversification Puzzle is Worse than you Think" American Economic Review, 87, 170-180.

[2] Campbell, J and L Viceira (2002) Strategic Asset Allocation Oxford, Oxford University Press.

[3] Devereux, M and M Saito (2005) "A Portfolio Theory of International Capital Flows" unpublished manuscript, UBC. 
[4] Devereux, M and A Sutherland (2007) "Country Portfolio Dynamics" CEPR Discussion Paper No 6208.

[5] Engel, C and A Matsumoto (2005) "Portfolio Choice in a Monetary Open-Economy DSGE Model" unpublished manuscript, University of Wisconsin and IMF.

[6] Evans, M and V Hnatkovska (2005) "International Capital Flows, Returns and World Financial Integration" NBER Working Paper 11701.

[7] Ghironi, F, J Lee and A Rebucci (2005) "The Valuation Channel of External Adjustment" unpublished manuscript, Boston College.

[8] Gourinchas, P and H Rey (2005) "International Financial Adjustment" NBER Working Paper no. 11155.

[9] Heathcote, J and F Perri (2004) "The International Diversification Puzzle is Not as Bad as You Think" unpublished manuscript, Georgetown University and New York University.

[10] Judd, K L (1998) Numerical Methods in Economics Cambridge: MIT Press.

[11] Judd, K L and S-M Guu (2001) "Asymptotic Methods for Asset Market Equilibrium Analysis" Economic Theory, 18, 127-157.

[12] Judd, K L, F Kubler and K Schmedders (2002) "A Solution Method for Incomplete Asset Markets with Heterogeneous Agents" unpublished manuscript, Stanford University.

[13] Kollmann, R (2006) "International Portfolio Equilibrium and the Current Account" unpublished manuscript, University of Paris XII.

[14] Lane, P, and G M Milesi-Ferretti (2001) "The External Wealth of Nations: Measures of Foreign Assets and Liabilities for Industrial and Developing Countries" Journal of International Economics 55, 263-94.

[15] Lane, P, and G M Milesi-Ferretti (2006) "The External Wealth of Nations Mark II" IMF Working Paper no 06-69. 
[16] Samuelson, P A (1970) "The Fundamental Approximation Theorem of Portfolio Analysis in terms of Means, Variances and Higher Moments" Review of Economic Studies, 37, 537-542.

[17] Schmitt-Grohe, S. and M. Uribe (2003) "Closing Small Open Economy Models", Journal of International Economics, 59, 137-59.

[18] Tille, C (2003) "The Impact of Exchange Rate Movements on U.S. Foreign Debt" Current Issues in Economics and Finance 9, 1-7.

[19] Tille, C (2004) "Financial Integration and the Wealth Effect of Exchange Rate Fluctuations" unpublished manuscript, Federal Reserve Bank of New York.

[20] Tille, C and E van Wincoop (2007) "International Capital Flows", NBER Working Paper No 12856. 